\section{Impulse aus der Forschung}

Mediative Kompetenzen bei Führungskräften ...260

Familienunternehmen scheitern an mangelnder Konfliktkompetenz ..................26r

\section{Im Fokus}

Elisabeth Kals

Anita von Hertel

Mediationskompetenzen ........... 262

Eine Einführung

Britt Füllenbach

Jens Rogmann

Alexander Redlich

Aspekte der Konfliktlösungskompetenz . . . . 270

Welche kommunikativen Fähigkeiten braucht

gute Konfliktbehandlung?

Heidi Ittner

Kompetenzen in der Mediation ...........280

Das Ganze ist mehr als die Summe seiner Teile

Anita von Hertel

Mediationskompetenz in der Praxis -

mit und ohne Mediator(in) . . . . . . . . . 286

So wirkt Mediationskompetenz vom ersten Schritt der Fallentwicklung bis zur nachhaltigen Umsetzung der Ergebnisse am Beispiel von »Frollegen(innen)《

\section{Theorie und Praxis}

\section{Rudolf Wimmer}

Macht und Organisation ............296

Über die Problematik des Machtbegriffs in der

Betrachtung von Organisationen heutigen Zuschnitts

Carmen Poszich-Buscher

Claudia Lutschewitz

Mediation mit Hirn ................. 306

Wie(so) Mediation auch aus neurobiologischer

Sicht ein wirkungsvolles Mittel zur Bearbeitung von Konflikten darstellt

\section{Brigitte Heidebrecht}

Tücken der Teamberatung ............ 314

Über Schwierigkeiten von Supervision bei Konflikten

in Teams und die Mediationsmethode Klärungshilfe

\section{Interview}

Das Kreativitätspotential organisationaler

Spannungsfelder nutzen ............. 323

Rudi Ballreich im Gespräch mit Rudolf Wimmer

\section{Rezensionen}

AUGENHÖHEwege (2016) . . . . . . . . . . . 329

Karl Kreuser, Volker Heyse, Thomas Robrecht (2012)

Mediationskompetenz - Mediation als Profession etablieren. Theoretischer Ansatz und zahlreiche

Praxisbeispiele .................... 330

\section{In Medias Res Juris}

\section{Renate Dendorfer}

»Entweder er oder wir« ............. 332

Druckkündigung oder Mediation als milderes Mittel?

\section{Kolumne}

Bernd Schmid

Schuld und systemisch? ............. 335

Kongresskalender .............. 331

Vorschau .........................336

Impressum ....................... 336 